\title{
Contexto y generalidades de la enseñanza del Canto como Licenciatura en tiempos de COVID-19
}

\section{Context and generalities of the teaching of Singing as a Bachelor in times of COVID-19}

\author{
CAIGNET, Solanye $\dagger^{*}$
}

Unidad de Artes de la Universidad Autónoma de Zacatecas, México.

ID $1^{\text {er }}$ Autor: Solanve, Caignet / ORC ID: 0000-0002-5559-2088, Researcher ID Thomson: ABI-6860-2020

DOI: $10.35429 / J P D .2020 .12 .4 .16 .21$

Recibido 20 de Enero, 2020; Aceptado 30 de Junio, 2020

Resumen

Objetivos:

- Contextualización de la educación vocal en forma virtual en situación de la pandemia.

- Descripción de la experiencia docente frente a las nuevas circunstancias de la educación vocal en línea

- Analizar los puntos de vista de algunos estudiantes de la Licenciatura en Canto sobre la Educación a distancia.

Metodología: La metodología utilizada es de tipo cualitativa exploratoria con investigación de campo bajo muestra no probabilística obtenida por consenso de los siete participantes de la encuesta, así como un seguimiento observacional basada en lo empírico en la enseñanza virtual de la materia de Canto-nivel Licenciatura.

Contribución:

- Revisión conceptual del tratamiento virtual en el área musical-vocal.

- Definición de antecedentes para formular una futura estrategia didáctica adecuada.

- Pre-diseño de una ruta académica que coadyuve a un mejor rendimiento para clases virtuales en el área vocal.

Clases virtuales, Pandemia, Enseñanza vocal, Rendimiento, Limitaciones

\begin{abstract}
Objectives:

- Contextualization of vocal education in virtual form in the situation of the pandemic.

- Description of the teaching experience in the face of the new circumstances of online vocal education

- Analyze the points of view of some students of the Bachelor of Singing on distance education.

Methodology: The methodology used is qualitative exploratory with field research under a non-probabilistic sample obtained by consensus of the seven participants in the survey, as well as an observational follow-up based on the empirical in virtual teaching of the subject of Bachelor-level Singing.

Contribution:

- Conceptual revision of the virtual treatment in the musical-vocal area.

- Definition of antecedents to formulate a future suitable didactic strategy.

- $\quad$ Pre-design of an academic route that contributes to a better performance for virtual classes in the vocal area.
\end{abstract}

Virtual classes, Pandemic, Vocal teaching, Performance, Limitations

Citación: CAIGNET, Solanye. Contexto y generalidades de la enseñanza del Canto como Licenciatura en tiempos de COVID-19. Revista de Didáctica Práctica. 2020. 4-11:16-21.

*Correspondencia al Autor (Correo Electrónico: solanyecl@uaz.udu.mx)

$\dagger$ Investigador contribuyendo como primer autor. 


\section{Introducción}

\section{Contexto actual}

La pandemia del COVID-19 originada en Wuhan, China, ha devastado a la población mundial, pues ha provocado infinidad de muertes y aún muchos más contagiados. Su impacto real aún está por revelarse. Los individuos forzados a utilizar cubrebocas, gel antibacterial y un frenético lavado de manos continúan navegando en un río de incertidumbre y nuestra esperanza de vida a la par de la pandemia es aún incierta. ""La gente tiene vidas más largas y saludables, lo malo es que el progreso es demasiado lento para lograr los Objetivos de Desarrollo Sostenible y que se retrocederá con el coronavirus" (Ghebreyesus, 2020)

En la cumbre mundial del 2015, se aprobó la Agenda 2030 para el Desarrollo sostenible. Entre sus objetivos está el de garantizar una educación inclusiva y equitativa de calidad y promover oportunidades de aprendizaje permanente para todos.

...asegurar que todos los alumnos adquieran los conocimientos teóricos y prácticos necesarios para promover el desarrollo sostenible... la valoración de la diversidad cultural y la contribución de la cultura al desarrollo sostenible. (Cepal, 2016).

Para mitigar los efectos de la pandemia los diferentes gobiernos e Instituciones se vieron forzados a detener y clausurar las actividades académicas en espacios comunitarios provocando una situación que afectaría al cien por ciento de la población docente y estudiantil. Debido al covid 19 las Instituciones educativas cierran sus puertas alrededor del 23 de Marzo de 2020. La educación impartida durante este año impone un antes y un después en el desarrollo pedagógico a nivel mundial. El impacto de la pandemia ha evidenciado la desigualdad social, cultural y económica en todos los países del mundo.
... hay entidades con muy bajo rezago social en los que la población puede acceder a mejores servicios de educación, de tal forma que, la mayoría de los hogares mexicanos se encuentran en condiciones de pobreza y casi una tercera parte de las entidades federativas presenta altos o muy altos grados de carencias sociales, lo que se ha traducido en la imposibilidad de muchos niños, niñas y jóvenes para dar continuidad a sus estudios desde casa. (Archundia, 2020)

Dentro de este contexto se ha desarrollado la educación desde el nivel básico hasta el nivel superior a través del sistema virtual de enseñanza. La educación musical, de manera online, en efecto ha constituido un paradigma aún no muy esclarecedor dentro de nuestras Instituciones artísticas. "Personalmente lo que más me preocupa es la pérdida de la vinculación social, presencial al hacer música." (Lagos, 2020)

La Unidad de Artes de la Universidad Autónoma de Zacatecas ha sido partícipe como el resto de las unidades de la nueva situación educativa dentro de la Institución, promoviendo y adecuándose a la situación pandémica. Docentes y alumnos, moldeándose a la nueva calendarización y organización que trajo como consecuencia el covid 19, han tenido que efectuar una enseñanza y aprendizaje estratégicos, pertinente e informativa a través de las diversas plataformas virtuales.

La tarea del docente se ha extendido y se ha complejizado. El docente tomaba en cuenta el contexto social y cultural del alumno dentro de la enseñanza para un mejor acercamiento y entendimiento de los contenidos académicos. Ahora la enseñanza virtual refleja un panorama que expone al docente $y$ al alumno en un ambiente complejo.

Me parece que al igual que en las otras áreas, hay un tema con la tecnología que está debilitando la frontera entre los mundos públicos y privados, en términos laborales y personales que está dañando enormemente lo que sucede en cada uno de esos espacios... porque son las profesoras y los profesores de música los que en este instante están siendo compelidos a tener que preparar una gran cantidad de clases. 
Muchos de ellos aprendiendo a usar sistemas que nunca han usado, lo que le quita horas a la familia, al descanso, a la producción personal y a la vez debilita incluso los aprendizajes que se puedan conseguir con los estudiantes. (Ibidem)

Así la enseñanza del Canto como Licenciatura subraya la importancia del contacto cercano entre docente y alumno debido a las características del aparato fonatorio. Este contacto propicia un trabajo de observación y análisis serio y contundente, debido a que el instrumento es el propio cuerpo y el principio de la voz como tal, se produce internamente. Las clases virtuales han tenido repercusiones negativas en el desarrollo de habilidades y en la situación emocional de los estudiantes de Canto: "el tiempo virtual puede generar distintas repercusiones en hábitos y costumbres cotidianas de los estudiantes, desde enfermedades leves hasta estrés". (De los Santos, 2020).

De este modo las obvias limitantes que se han desarrollado también han desatado la creatividad y el ingenio tanto de docentes como alumnos para una integralidad educativa a distancia y en línea.

De esta manera lo que esta investigación persigue es una descripción del panorama actual de la enseñanza virtual que se ha tenido que llevar a cabo para impartir clases en la Licenciatura en Canto de la Unidad de Artes de la Universidad Autónoma de Zacatecas desde las dos perspectivas involucradas: la experiencia docente y la visión del alumno. La contribución más importante que se pretende es mantener un seguimiento metodológico como fundamento para el futuro progreso de la enseñanza vocal en los medios virtuales.

Por consiguiente, la presente investigación se ha dividido en varias secciones que van desde la contextualización de la situación pandémica y su afectación, así como la problematización de impartir clases virtuales en el área del Canto.

Los objetivc
investigación son:

Contextualización de la educación virtual en situación de la pandemia.
- Descripción de la experiencia docente frente a las nuevas circunstancias de la educación en línea, u online.

- Analizar los puntos de vista de algunos estudiantes de la Licenciatura en Canto sobre la

- Educación a distancia.

La metodología atributiva explora dentro de dos acercamientos prioritarios ambas visiones.

\section{Enseñanza del canto en tiempos pandémicos}

La educación a distancia presenta límites de cobertura, pues se dificulta el seguimiento del aprendizaje práctico. Los contenidos a vencer dentro de la clase de canto son diversos, las habilidades que se desarrollan, generalmente tienen que ver con la correcta coordinación muscular y el desarrollo auditivo, así como capacidades actorales que involucran lo corpóreo.

Mantenerse en línea exige recursos económicos, algunos estudiantes no cuentan ni siquiera con una computadora para llevar a cabo sus actividades académicas. El estudio de la música a través de los medios virtuales ha tomado nuevos rumbos $\mathrm{y}$ presenta un paradigma complejo que se ha ido develando a través de la pandemia actual.

La UNESCO ofrece recomendaciones específicas para el estudio en tiempo de pandemias (ONU, 2020):

- Analizar el tipo de tecnología con el que se cuenta y escoger la más pertinente.

- $\quad$ Proteger la privacidad y seguridad de los datos.

Concentrarse en los problemas psicosociales del alumnado antes de impartir la enseñanza.

- Planificar con frecuencia el desarrollo de los programas de aprendizaje online.

- Proporcionar a docentes y alumnado asistencia en cuanto al uso de las TICs. 
Establecer las reglas de la educación online y dar seguimiento al proceso de aprendizaje.

- Definir el tiempo de duración de las clases online en función del alumnado.

- Crear comunidades entre el profesorado y favorecer los vínculos sociales.

El reto de la educación virtual ha sido mantener la continuidad de la educación, así como promover aprendizajes significativos. Desde hace décadas se hablaba de las clases virtuales como una estrategia complementaria a las clases presenciales. Las TIC's representaban herramientas al servicio de la educación.

La educación virtual no necesita un espacio o tiempo específicos y esto abre un nuevo horizonte comunicativo entre docentes y alumnos.

¿Que se requiere para una efectiva educación virtual?

- $\quad$ Adecuados recursos tecnológicos.

- $\quad$ Acceso a la plataforma educativa en uso.

- Que los contenidos ofrezcan al alumno la información necesaria.

- Aprendizajes efectivos y un ambiente adecuado para el docente y para el alumno.

Así las plataformas que rara vez se utilizaban antes, en tiempos del COVID-19 están rebasadas y trabajando al máximo de su capacidad. Las redes sociales han ganado valor como recurso académico pero la pandemia ha maximizado ese valor, a tal punto que hoy en día se utilizan a la par de las plataformas educativas.

\section{Experiencia personal como docente de la materia}

El rigor de las clases presenciales se pierde y afecta el compromiso que se tiene frente a la enseñanza, al no estar el docente ni el alumno forzados a un horario, se flexibiliza el manejo de los horarios virtuales.
La viabilidad de la señal de internet puede ser muy variable durante el día, por lo que las clases se pueden ver afectadas por la falta de señal. La visión por medio de la pantalla es incompleta e ineficiente. El otro elemento imprescindible es el sonido que es escaso para el trabajo vocal por las dimensiones de las voces. También se pueden presentar continuas interferencias con el audio. Esto ha provocado riesgos de trabajo que afectan directamente al instrumento vocal, pues resulta común alzar la voz para el entendimiento entre las partes, así como graves problemas físicos desde cervicales dañadas o problemas de columna en general. Siendo la postura física vital en la clase de canto, se comienzan a generar problemas posturales que afectan el desarrollo de las habilidades.

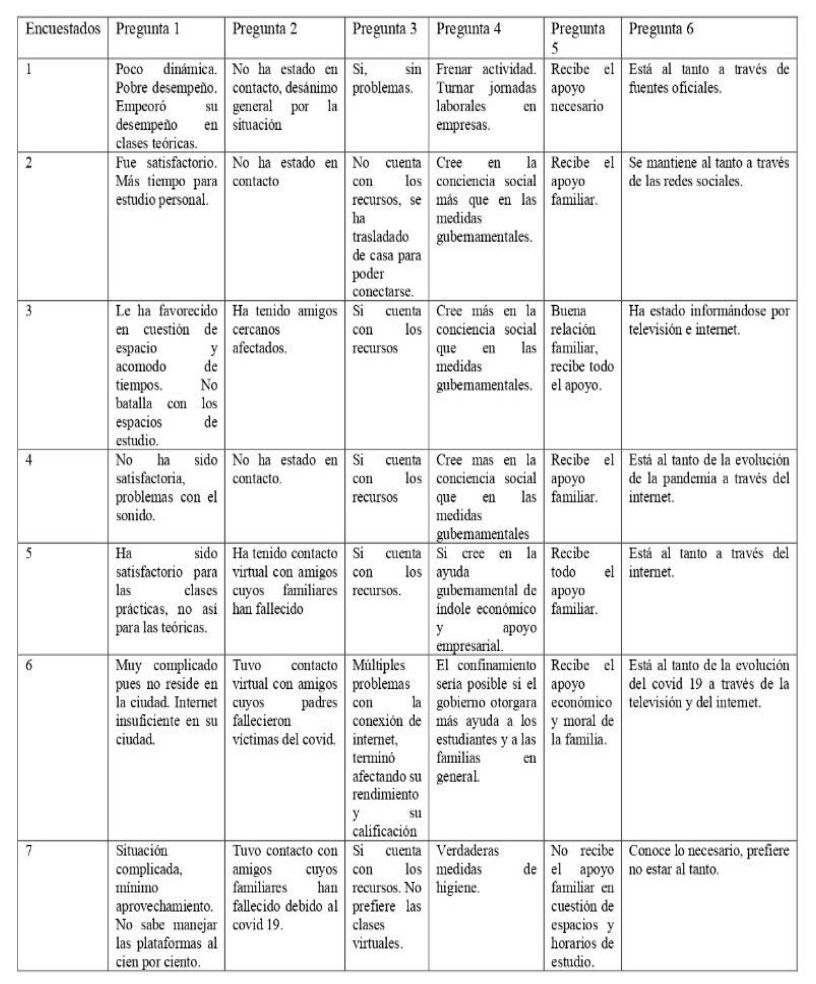

Tabla 1

\section{Metodología}

Esta es una investigación cualitativa, de enfoque interpretativo con investigación de campo con muestra probabilística por el consenso de los siete encuestados.

La primera parte está basada en una metodología empírica y observacional basada en la propia experiencia como docente de canto en la actual situación pandémica. 
La segunda parte está basada en la investigación de campo realizada a través de una encuesta descriptiva de respuesta abierta de siete estudiantes que cursan la Licenciatura en Canto obteniendo así un pronóstico más profundo en cuanto al tema del funcionamiento de su experiencia virtual en la actualidad.

\section{Resultados}

Acorde a la reciente experiencia como docente, las situaciones específicas que se han suscitado con las clases virtuales para la enseñanza del canto han sido las siguientes:

- Visión limitada, por lo que no se puede tener una completa referencia de la coordinación muscular que el alumno emplea en su emisión vocal.

- $\quad$ Sonido limitado. Al ser la voz impostada un tipo de voz para espacios grandes, se produce una sobresaturación del micrófono de la computadora.

- Problemas posturales, tanto para el docente como para el alumno cuando se produce un acercamiento a la pantalla.

- $\quad$ Espacio limitado por lo que el alumno se siente en un ambiente un poco opresivo en cuánto a su interpretación.

- Aprendizaje por parte de docentes y alumnos sobre el uso de ciertas plataformas, lo que se traduce en tiempo y disponibilidad.

Imposibilidad de dejar la clase grabada con antelación pues al ser una clase práctica es imprescindible el contacto en tiempo y forma.

La encuesta realizada se elaboró con las siguientes preguntas:

1- ¿Como ha sido tu experiencia con la actual situación de clases virtuales en tu entorno educativo?

2- Si ha estado en contacto con la muerte de algún familiar o conocido, responde por favor: ¿de qué manera te ha impactado psicológicamente?
3- ¿Cuentas con los recursos para mantenerte en línea buena parte del día y recibir tus clases virtuales?

4- ¿Qué medidas crees que el gobierno necesita implantar con la actual situación del COVID-19?

5- ¿Qué relación ha creado con tu familia la actual situación? ¿Te han apoyado en tu educación virtual?

6- ¿Estás al tanto de las nuevas noticias sobre el COVID-19? ¿Por qué medio? ¿Qué sabes del virus?

Las respuestas de los encuestados fueron las siguientes: (Respuestas en la Tabla)

\section{Annexes}

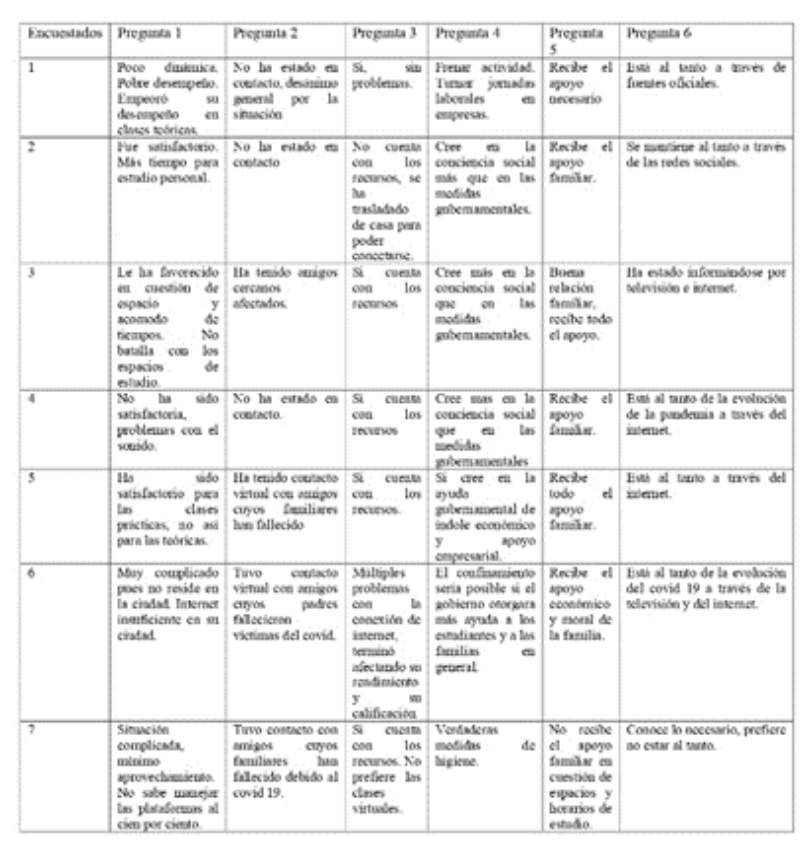

Tabla 2

\section{Agradecimientos}

Esta investigación no ha sido financiada por ninguna Institución.

\section{Conclusiones}

La pandemia del COVID-19 ha creado una relación diferente en las dinámicas académicas en los estudiantes de Canto de la Licenciatura de la unidad de Artes de la UAZ. Esto puede ser relativo en base a los diferentes accesos tecnológicos y el uso de micrófonos adecuados. 
La enseñanza del instrumento vocal se ha transformado en las clases virtuales y requiere de una visión estabilizadora que constantemente aporte elementos positivos para su función.

La misma flexibilidad entre horarios y el problema de los espacios desarrolla un desalentador panorama para el estudiante pues se puede magnificar la falta de interés por tomar la clase virtual de canto. Los alumnos pueden también sentirse desalentados por la falta de contacto físico con sus compañeros de clase, así como la falta de instrumentos necesarios como el piano dentro de su ambiente escolar.

Aun así, reconocen que la Institución trató de solventar la continuidad del Semestre académico dentro de los parámetros impuestos por la pandemia, y obtuvieron un resultado final aunado a la calificación.

Los tiempos del COVID-19 ha puesto en evidencia la inclusión de los diferentes medios del aprendizaje y la necesaria transformación educativa. El uso de las plataformas virtuales, así como la manera de minimizar los riesgos físicos de trabajo es una constante que debe de ser analizada por medio del continuo seguimiento de la enseñanza y aprendizaje en línea, que se ha visualizado como necesaria para el crecimiento de los parámetros educativos dentro de la Institución.

\section{Referencias}

OMS, 2020, Coronavirus amenaza con reducir esperanza de vida global, Milenio Diario S.A de C.V., En línea https://www.milenio.com/internacional/coronav irus-amenaza-reducir-esperanza-vida-advierteoms

Naciones Unidas (2018), La Agenda 2030 y los Objetivos de Desarrollo Sostenible: una oportunidad para América Latina y el Caribe (LC/G.2681-P/Rev.3), Santiago. En línea https://repositorio.cepal.org/bitstream/handle/11 362/40155/24/S1801141_es.pdf

Poblete Carlos, (2020), La educación musical en tiempos de pandemia, ¿diversidad o diferencia cultural?, Entrevistado por la revista Atemus.
Medina Ana, (Junio, 2020), La covid-19, transformación educativa obligada, Paraguay 2020, Academic Disclosure, Spetial edition covid-19, num 1, (p. 40-51), Recuperado de: https://revistas.fenob.una.py/ojs/index.php/rfen ob/article/view/68/13

Mansilla Alan M., (Diciembre, 2020), Impacto del covid-19 en los hábitos y costumbres de estudiantes universitarios durante el tiempo de cuarentena 2020, Revista Hacedor, num 2, (p.111), Recuperado de: http://revistas.uss.edu.pe/index.php/HACEDOR /article/view/1478

ONU, (Marzo, 2020), Diez recomendaciones para estudiar a distancia durante la emergencia del coronavirus, URL: https://news.un.org/es/story/2020/03/1471342

Rogero-Garcìa Jesùs., (Abril, 2020), La ficción de educar a distancia, RASE, num 2, (p. 174182), Recuperado de: https://ojs.uv.es/index.php/RASE/article/view/1 $7126 / 15397$. 